\title{
CARACTÉRISATION EXPÉRIMENTALE DE BRIQUETTES À BASE DE DÉCHETS PAPIERS/CARTONS ET ÉTUDE DE L'IMPACT DU LIANT LORS DE LEUR CONCEPTION
}

\author{
Baguian Abdoul Fayçal ${ }^{1}$, Ouiminga Salifou Koucka ${ }^{1,}$ ", Gado Ibrahim Harouna ${ }^{2}$, \\ Sidibé Sayon Sadio ${ }^{3}$, and Béré Antoine ${ }^{1}$ \\ ${ }^{I}$ Laboratoire de Physique et de Chimie de l'Environnement, Université Joseph KI-ZERBO, Ouagadougou, Burkina Faso \\ ${ }^{2}$ Facultés des Sciences et Techniques, Université Dan Dicko Dankoulodo de Maradi, Niger \\ ${ }^{3}$ Laboratoire Biomasse Énergie Biocarburant, Institut International d'Ingénierie de l'Eau et de l'Environnement (2iE), \\ Ouagadougou, Burkina Faso \\ *Email : salif0477@yahoo.com
}

\author{
INFOS SUR L'A R T I C L E \\ Historique de l'article: \\ Reçu le : 26 novembre 2020 \\ Reçu en format revisé le 27 juin 2021 \\ Accepté le : 29 juin 2021
}

Mots-Clés : briquettes de combustibles ;

déchets papier/carton; relaxation ;

densité ; $P C I$

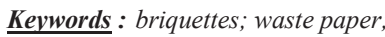
relaxation; density; Calorific value

\section{INTRODUCTION}

La gestion des déchets est une préoccupation dans la plupart de nos villes. Toutefois, pour booster les secteurs préoccupants présentés par nos décideurs, à savoir l'éducation, la santé, la fourniture d'eau potable, l'agriculture, la construction des infrastructures, nous avons besoin de travailler dans un environnement sain, en l'occurrence un milieu ambiant avec un système de gestion optimal des déchets. Ainsi, les déchets devraient faire

\begin{abstract}
R E S U M E
Nous avons étudié la faisabilité technique de la valorisation des déchets papiers/cartons pour la fabrication des briquettes combustibles. L'objectif de cette étude est de contribuer à la valorisation matière de la filière déchets ménagers, précisément celle des déchets papiers/cartons. Pour l'aspect technique, l'étude a été faite sur l'impact de la gomme arabique et la mélasse sur la qualité des briquettes à travers l'analyse de leurs caractéristiques physiques (indice de résistance l'impact, taux de relaxation, densité) et thermiques (taux d'humidité, taux de matières volatiles, taux de cendre, taux de carbone fixe, pouvoir calorifique inferieur) pour connaître les briquettes de meilleure qualité. La pression de compactage était à 60bars. Ces analyses nous ont permis de mettre en évidence trois types de briquettes de bonne qualité. Il s'agit de celles issues des mélanges avec la gomme arabique (50\% de déchets papiers et $50 \%$ de gomme arabique), celles issues des mélanges avec la mélasse ( $50 \%$ de déchets papiers et $50 \%$ de mélasse) et celles issues exclusivement des déchets papiers compactés directement avec l'eau (50\% de déchets papiers et $50 \%$ d'eau). Toutes les briquettes produites à partir du papier ont un Pouvoir Calorifique Inférieur de l'ordre de $14 \mathrm{MJ} / \mathrm{kg}$ quel que soit le mélange papiers/cartons-liant testé ; avec cependant un PCI plus élevé pour les briquettes contant de la mélasse comme liant. Les résultats obtenus lors de nos travaux nous permettent de conclure que la mélasse est le meilleur liant pour la cohérence des briquettes, pour une faible masse volumique des briquettes; aussi elle permet d'avoir un meilleur IRI des briquettes, et leur assure une meilleure durabilité. Concernant les briquettes elles-mêmes, on peut affirmer que les briquettes fabriquées avec l'eau présentent un faible taux d'humidité par rapport à celles fabriquées avec la gomme arabique et la mélasse, tandis que les briquettes fabriquées avec la gomme arabique possède un plus faible taux de matières volatiles. Pour finir, d'autres résultats nous montrent un taux de cendre qui varie très peu pour les briquettes fabriquées avec la mélasse et un taux de carbone fixe élevé dans celles conçues avec de l'eau.
\end{abstract}

l'objet d'une attention particulière (BEMB G. C., 2009). La gestion des déchets, particulièrement celle des déchets solides constitue l'un des défis de la plupart des collectivités territoriales surtout pour les moyennes et grandes villes où la croissance démographique et la diversité des activités socio-économiques sont à l'origine de la dégradation de l'environnement. Les ordures ménagères produites à Ouagadougou contiennent une part non négligeable de papiers et de cartons de l'ordre de $10 \%$ soit 20000 tonnes par an (GADO I. H. et al. 2013). Un bon 
système de gestion de ces déchets constituant un créneau indispensable de mettre en place un plan de gestion des déchets (DUSABE M. S., 2014). Une des actions de ce plan de gestion serait la valorisation matière, le recyclage et ou la réutilisation des déchets qui peuvent l'être. Parmi ces déchets valorisables, nous pouvons citer le papier et les cartons communément appelé papiers/cartons (Mélanie PROUST, 2001). Plusieurs types de traitement pour ces papiers/cartons existent. Une grande partie est potentiellement valorisable et ce, en fonction de leur nature chimique, de leurs propriétés mécaniques, physicochimiques ou de leur pouvoir calorifique inférieur (PCI). Aussi, la pénurie actuelle des énergies traditionnelles au Burkina Faso (bois et charbon du bois) entraine un sérieux problème de déboisement et donc d'érosion et d'appauvrissement des sols, d'approvisionnement, de désertification et des hausses des prix (DUSABE M. S., 2014). Toute chose qui montre la nécessité de la valorisation des déchets notamment la transformation des déchets papiers/cartons en briquettes qui pourront éventuellement se substituer partiellement aux combustibles traditionnels (bois, charbons...). Cette dernière voie proposée est l'objet de notre étude étant donné que les briquettes à base de papier/carton sont une source potentielle d'énergie renouvelable si elles sont fabriquées à partir de la biomasse (Njenga, M. et al. 2009). Afin de rendre beaucoup plus cohérent, rigide et stable ces briquettes, nous testerons deux types de liants à savoir la gomme arabique et la mélasse qui présentent ces caractéristiques. Si une telle solution était envisagée elle permettrait de réduire les nuisances liées à la pollution environnementale engendrée par ce type de déchets et de baisser sensiblement le coût de transport de ces déchets. Un autre avantage serait la proposition de solutions aux autorités du Burkina Faso qui sont aujourd'hui prêtes à investir dans d'autres sources alternatives de combustibles à même de se substituer partiellement ou totalement au bois de chauffage et au charbon du bois. L'objectif principal est de contribuer à la valorisation matière de la filière déchets ménagers, précisément celle des déchets papiers/cartons. Plus spécifiquement, il s'agit (i) de concevoir avec deux types de liants des briquettes à base de papiers/cartons, (ii) d'étudier l'impact du liant sur la qualité des briquettes à travers l'analyse de leurs caractéristiques physiques, mécaniques et chimiques.

\section{MATERIELS ET METHODES}

\subsection{Matériels}

\subsubsection{Choix des matières premières}

La collecte des déchets papiers/cartons s'est faite au sein de l'Université Ouaga I Pr Joseph KI-ZERBO à l'aide de poubelles que nous avons positionnées devant les salles de classe. La gomme arabique et la mélasse ont été achetées respectivement à Ouagadougou et à la Société Sucrière de la Comoé (SOSUCO) à Banfora.

\subsubsection{Dispositif expérimental}

Le briquetage et les analyses physiques, thermiques et mécaniques ont été effectués à l'aide du dispositif comprenant un déchiqueteur pour déchiqueter les papiers/cartons en morceaux de petites tailles (inférieur à $5 \mathrm{~mm}$ de diamètre) afin d'optimiser l'homogénéisation des briquettes. La balance de précision (1 $\mathrm{mg}$ au moins) qui servira pour la mesure de la masse des échantillons de déchets papiers/cartons et des liants. Une presse manuelle (pression maximale de 100 bars) pour le compactage des déchets papiers/cartons. Un pied à coulisse précision de $0,02 \mathrm{~mm}$ pour mesurer la taille des briquettes afin de déduire la masse volumique et le taux de relaxation des briquettes. Une étuve de marque Heraeus utilisée comme séchoir pour les briquettes fabriquées et la détermination du taux d'humidité des briquettes. Un dispositif (une pince accrochée à un fer) pour maintenir la briquette à $2 \mathrm{~m}$ du sol afin de mesurer sa résistance à l'impact. Un four à moufle programmable pour la mesure du taux de matières volatiles, du taux de cendres et du taux de carbone fixe. Une bombe calorimétrique pour la mesure du PCI.

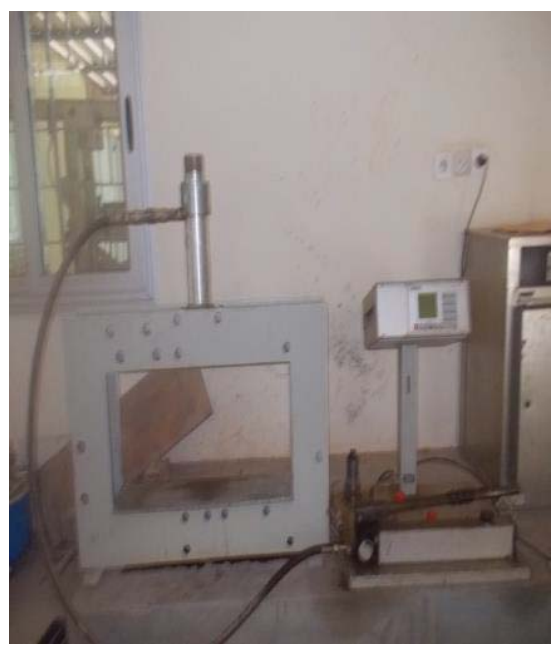

Fig.1. Presse hydraulique manuelles

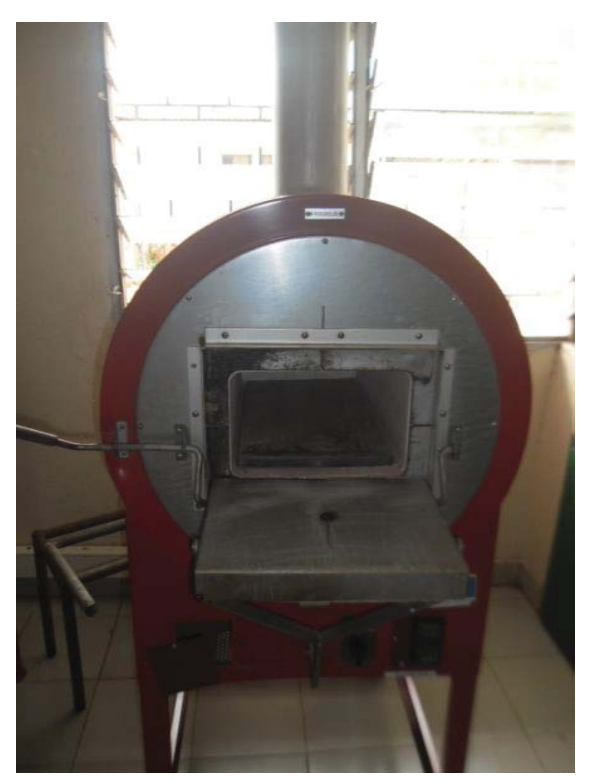

Fig.2. Four à moufle 


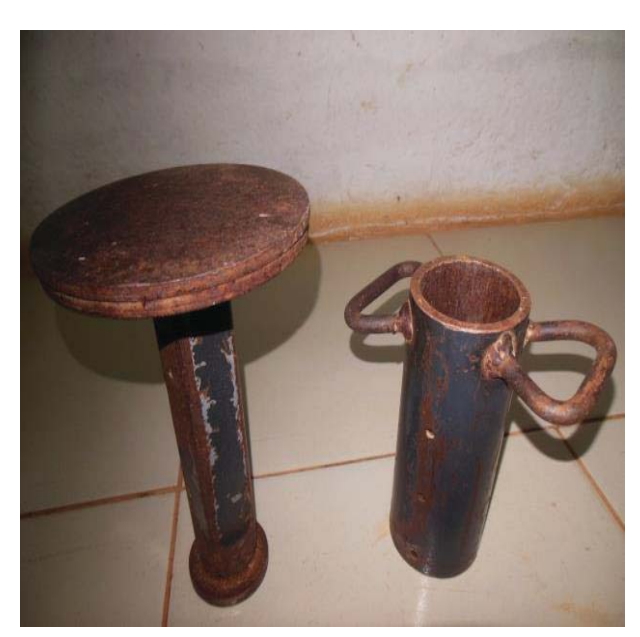

Fig.3. Moule pour briquetage

\subsection{Méthodes}

\subsubsection{Protocole expérimental}

D'après les travaux d'Ibrahim H. GADO et al. (2013), 60 bars est la pression optimale de compactage de tous types de briquettes à base de déchets papiers/cartons. La masse d'échantillon par essai (déchets papiers + liant) est de 100 grammes et est compactée à une pression de 60 bars. La gomme a été concassée en de petits morceaux à l'aide d'un mortier. Pour chaque liant nous avons trois mélanges distincts pour la fabrication des briquettes :

Le tableau 1 montre les proportions des matières premières utilisées pour le briquetage.

Table 1. Choix de la composition

\begin{tabular}{|l|l|}
\hline $\begin{array}{l}\text { Nom des } \\
\text { briquettes }\end{array}$ & Proportions des matières premières \\
\hline P90\%G10\% & $\begin{array}{l}\text { déchets papier }(90 \mathrm{~g})+\text { gomme } \\
\text { arabique }(10 \mathrm{~g})\end{array}$ \\
\hline $\mathrm{P} 70 \% \mathrm{G} 30 \%$ & $\begin{array}{l}\text { déchets papier }(70 \mathrm{~g})+\text { gomme } \\
\text { arabique }(30 \mathrm{~g})\end{array}$ \\
\hline $\mathrm{P} 50 \% \mathrm{G} 50 \%$ & $\begin{array}{l}\text { déchets papier }(50 \mathrm{~g})+\text { gomme } \\
\text { arabique }(50 \mathrm{~g})\end{array}$ \\
\hline $\mathrm{P} 90 \% \mathrm{E} 10 \%$ & déchets papier $(90 \mathrm{~g})+$ eau $(10 \mathrm{~g})$ \\
\hline $\mathrm{P} 70 \% \mathrm{E} 30 \%$ & déchets papier $(70 \mathrm{~g})+$ eau $(30 \mathrm{~g})$ \\
\hline $\mathrm{P} 50 \% \mathrm{E} 50 \%$ & déchets papier $(50 \mathrm{~g})+$ eau $(50 \mathrm{~g})$ \\
\hline $\mathrm{P} 90 \% \mathrm{M} 10 \%$ & déchets papier $(90 \mathrm{~g})+$ mélasse $(10 \mathrm{~g})$ \\
\hline $\mathrm{P} 70 \% \mathrm{M} 30 \%$ & déchets papier $(70 \mathrm{~g})+$ mélasse $(30 \mathrm{~g})$ \\
\hline $\mathrm{P} 50 \% \mathrm{M} 50 \%$ & déchets papier $(50 \mathrm{~g})+$ mélasse $(50 \mathrm{~g})$ \\
\hline
\end{tabular}

\subsubsection{Paramètres physiques, thermiques et mécaniques}

La performance des briquettes est déterminée à partir des paramètres tels que le PCI, la masse volumique, la résistance à l'impact, la relaxation, le taux d'humidité, le taux de matières volatiles, le taux de cendre, le taux de carbone fixe. L'objectif est de déterminer le mélange optimal pour la conception de briquettes et l'impact des liants utilisés sur la qualité des briquettes fabriquées.

a) Le Pouvoir calorifique inférieur (PCI)

Principe de mesure : Le PCI est déterminé à l'aide d'une bombe calorimétrique en présence d'oxygène et un suivi de température toutes les 30 secondes doit être assuré jusqu'à la stabilisation. Sa détermination suit la norme française NF ISO 1928, 2004. Cette méthode permet de déterminer le Pouvoir Calorique Supérieur (équation 1) et le PCI est déduit par simple calcul à partir du PCS (équation 2).

$$
\begin{aligned}
& \text { PCS }=\frac{K_{1} * E_{c a l} *\left(T_{m}-T_{i}\right)-K_{1} * E_{p t} *\left(L_{i}-L_{f}\right)}{m_{\text {echantillon }}} \\
& \text { PCI }=\text { PCS }-\left(\frac{K_{1} * E_{\text {cond }} * H_{\text {ech }}}{100}+\frac{E_{c o n d} * W}{100}\right)
\end{aligned}
$$

b) La masse volumique ( $\rho$ )

Les dimensions de la briquette sont mesurées par un compas. La Norme AFNOR/X 34 B. N-287 donne le calcul de la masse volumique par l'équation (3) :

$\rho=\frac{m}{V} \quad$ avec $V=\frac{\mathbf{D}^{2} * \boldsymbol{\pi} * \mathbf{H}}{4}$

$\mathrm{D}$ : le diamètre, $\mathrm{H}$ : la hauteur, $\mathrm{V}$ : le volume et $\mathrm{m}:$ la masse de la briquette.

\section{c) L'indice de résistance à l'impact (IRI)}

Pour determiner la resistance à l'impact d'une briquette, on place un dispositif à une hauteur de 2 mètres du sol et on laisse chutter la briquette. Cela se repète jusqu'à ce que la briquette se casse. Cette experimentation se fait avec des briquettes de même type et de la même pression. D'après Richards (1990), l'equation (4) de la resistance à l'impact est :

$$
R I=\frac{100 * N}{n}
$$

$n$ : le nombre de morceaux de briquette après la cassure, $N$ : le nombre de chute de la briquette (Richards S. R., 2001).

\section{d) Le taux de relaxation (\%li)}

Après avoir fini la fabrication de la briquette, sa longueur a tendance à s'augmenter avec le temps. Elle passe d'une longueur $l_{0}$ à une longueur maximale $1_{\max }$ avant de se stabiliser. $1_{0}$ est la longueur de la briquette immédiatement après sa sortie du moule; $1_{\max }$ est la longueur maximale atteinte par la briquette dix minutes après sa sortie du moule. Selon les travaux de Chin, O. C., généralement le maximum de relaxation dure dix minutes et nous avons pu le vérifier sur nos briquettes produites. Le maximum de relaxation est donné par l'équation (5) :

$$
\begin{aligned}
& \% \mathrm{l}_{\mathrm{i}}=100 * \frac{\left(\mathrm{l}_{\mathrm{max}}-\mathrm{l}_{0}\right)}{\mathrm{l}_{\mathrm{o}}} \\
& \text { e) Le taux d'humidité }(\mathrm{W})
\end{aligned}
$$

C'est un paramètre déterminant pour la combustion des briquettes : s'il est élevé, la combustion est quasiimpossible. 
Principe de mesure : L'échantillon est placé dans une étuve à $105^{\circ} \mathrm{C}$ pendant 2 heures et on effectue une pesée après l'avoir sorti. Sa détermination suit la norme européenne Norme AFNOR/X 34. Le taux d'humidité est obtenu par la formule suivante (6) :

$\mathrm{W}(\%)=100 * \frac{\mathrm{m}_{2}-\mathrm{m}_{3}}{\mathrm{~m}_{3}-\mathrm{m}_{1}}$

W : taux d'humidité, $\mathrm{m}_{2}$ : masse de l'échantillon + la coupelle, $\mathrm{m}_{3}$ : masse obtenu après chauffage à $105^{\circ} \mathrm{C}$ à l'étuve, $\mathrm{m}_{1}$ : masse de la coupelle.

\section{f) Le taux de matières volatiles (MOV)}

D'après la norme française MO3-004, pour les papiers/cartons la température à laquelle les matières volatiles sont obtenues est de $550^{\circ} \mathrm{C}$. Le taux de $\mathrm{MOV}$ cautionne l'inflammabilité du combustible.

Principe de mesure : Le même échantillon utilisé pour trouver le taux d'humidité est chauffé dans un four à une température allant jusqu' à $550^{\circ} \mathrm{C}$. Sa détermination suit la norme française NF MO3-004 et internationale ISO 562.

Le taux de matières volatiles est déterminé par la perte de masse pendant ce chauffage. La formule suivante permet de calculer le taux de matières volatiles (7):

MOV $=100 * \frac{\mathrm{m}{ }_{2}-\mathrm{m} / 3}{\mathrm{~m} \prime_{2}-\mathrm{m} \prime_{1}}$

MOV : Taux de matières volatiles,

$\mathrm{m}_{1}^{\prime}$ : masse du creuset vide muni de son couvercle,

$\mathrm{m}_{2}^{\prime}$ : masse du creuset + de l'échantillon + le couvercle,

$\mathrm{m}_{3}^{\prime}$ : masse $\mathrm{du}$ creuset + résidu + couvercle après chauffage à $900^{\circ} \mathrm{C}$ pendant sept (7) minutes.

g) Le taux de cendres (A)

Le taux de cendres représente la quantité des matières minérales contenues dans un combustible. Il est important pour l'appréciabilité du combustible du fait que quand il est très élevé, ces cendres deviennent obstacle à la progression de la combustion.

Principe de mesure : Le taux de cendres est obtenu par le chauffage de l'échantillon jusqu'à $815^{\circ} \mathrm{C}$ dans un four moufle. Sa détermination suit la norme européenne EN 14775. Le taux de cendres est déterminé par la masse des résidus après incinération. Le résultat est obtenu avec la formule suivante (8) :

$A(\%)=100 * \frac{\mathrm{m} \prime_{3}-\mathrm{m} \prime_{1}}{\mathrm{~m} \prime_{2}-\mathrm{m} \prime_{1}}$

A : taux de cendres,

$\mathrm{m}_{3}^{\prime \prime}$ : masse de la poudre obtenue après incinération à $815^{\circ} \mathrm{C}$,

$\mathrm{m}_{2}^{\prime \prime}$ : masse de l'échantillon + creuset,

$\mathrm{m}^{\prime \prime}{ }_{1}$ : masse du creuset vide.

h) Le taux de carbone fixe (CF)

C'est la quantité restante de carbone après élimination des matières volatiles, des cendres et de l'humidité. Il est différent de carbone total qui est la somme du carbone fixe et le carbone contenu dans la partie volatilisée. Le taux de carbone fixe est déterminé en suivant la norme ASTM et il est calculé par la formule suivante (9) :
$\mathrm{CF}(\%)=100-\operatorname{MOV}(\%)-\mathrm{A}(\%)$

\section{RESULTATS ET DISCUSSION}

Cette partie de l'étude a pour but de présenter les résultats auxquels notre travail a abouti. Nous présentons les résultats de l'étude de faisabilité technique. Ces résultats seront accompagnés par leurs interprétations et discussion.

\subsection{Résultats obtenus}

Le taux d'humidité présenté est le pourcentage de la teneur en eau du combustible à l'état naturel (matière humide). Par contre, les valeurs des quatre paramètres (taux de matières volatiles, taux de cendres, le taux de carbone fixe et le $\mathrm{PCI}$ ) présentées ont été analysées sur la matière sèche. Les résultats détaillés sont présentés dans les tableaux 2.a et 2.b.

Table.2.a. Résultat des tests

\begin{tabular}{|l|c|c|c|c|}
\hline Briquettes & $\begin{array}{c}\text { Relaxation } \\
\text { en \% }\end{array}$ & $\begin{array}{c}\text { Masse } \\
\text { volumique } \\
\text { g/cm3 }\end{array}$ & IRI & $\begin{array}{c}\text { W en } \\
\%\end{array}$ \\
\hline P90\%G10\% & 5,2 & 0,879 & 800 & 8,4 \\
\hline P70\%G30\% & 4,79 & 0,721 & 1000 & 9,6 \\
\hline P50\%G50\% & 2,38 & 0,721 & 1000 & 11,4 \\
\hline P90\%E10\% & 40,32 & 0,675 & 144,4 & 8,1 \\
\hline P70\%E30\% & 16,6 & 0,663 & 291,6 & 8,3 \\
\hline P50\%E50\% & 12,78 & 0,628 & 1000 & 9,2 \\
\hline P90\%M10\% & 4,23 & 0,649 & 272,2 & 8,5 \\
\hline P70\%M30\% & 2,87 & 0,544 & 600 & 9,4 \\
\hline P50\%M50\% & 1,53 & 0,54 & 1000 & 15,1 \\
\hline $\begin{array}{l}\text { Déchet } \\
\text { papier }\end{array}$ & - & - & - & 7,3 \\
\hline $\begin{array}{l}\text { Gomme } \\
\text { arabique }\end{array}$ & - & - & - & 12,3 \\
\hline Mélasse & - & - & - & 26,9 \\
\hline
\end{tabular}

Table.2.b. Résultat des tests

\begin{tabular}{|l|c|c|c|c|}
\hline Briquettes & $\begin{array}{c}\text { MOV } \\
\text { en \% }\end{array}$ & $\begin{array}{c}\text { A en } \\
\%\end{array}$ & $\begin{array}{c}C f \text { en } \\
\%\end{array}$ & $\begin{array}{c}\text { PCI en } \\
\text { MJ/kg }\end{array}$ \\
\hline P90\%G10\% & 77,7 & 9,2 & 13,1 & 12,9 \\
\hline P70\%G30\% & 76,9 & 8,8 & 14,3 & 13,6 \\
\hline P50\%G50\% & 76,4 & 7,9 & 15,6 & 13,9 \\
\hline P90\%E10\% & 77,4 & 11,1 & 11,4 & 13,4 \\
\hline P70\%E30\% & 74,7 & 11,5 & 13,8 & 13,2 \\
\hline
\end{tabular}




\begin{tabular}{|l|c|c|c|c|}
\hline Briquettes & $\begin{array}{c}\text { MOV } \\
\text { en \% }\end{array}$ & $\begin{array}{c}\text { A en } \\
\%\end{array}$ & $\begin{array}{c}\text { Cf en } \\
\%\end{array}$ & $\begin{array}{c}\text { PCI en } \\
\text { MJ/kg }\end{array}$ \\
\hline P50\%E50\% & 70,6 & 11,6 & 17,7 & 13,2 \\
\hline P90\%M10\% & 80 & 11 & 8,9 & 13,1 \\
\hline P70\%M30\% & 81,5 & 10,8 & 7,6 & 13,7 \\
\hline P50\%M50\% & 82,3 & 10,5 & 7,1 & 14,3 \\
\hline Déchet papier & 79,4 & 11,8 & 8,7 & 12,7 \\
\hline Gomme arabique & 75,3 & 2,3 & 22,3 & 15,2 \\
\hline Mélasse & 84,5 & 5,3 & 10,1 & 15,6 \\
\hline
\end{tabular}

3.2. Impact de la gomme arabique et de la mélasse sur le compactage

\subsubsection{Le taux de relaxation}

Une des caractéristiques importantes d'une briquette est son taux de relaxation. Le liant joue un rôle très primordial dans la relaxation de la briquette parce qu'il facilite la cohésion des déchets papiers déchiquetés. Nous avons un taux de relaxation très variable ( 1 à $40 \%$ ) en fonction du type et des proportions du liant comme le montre la Figure 4.

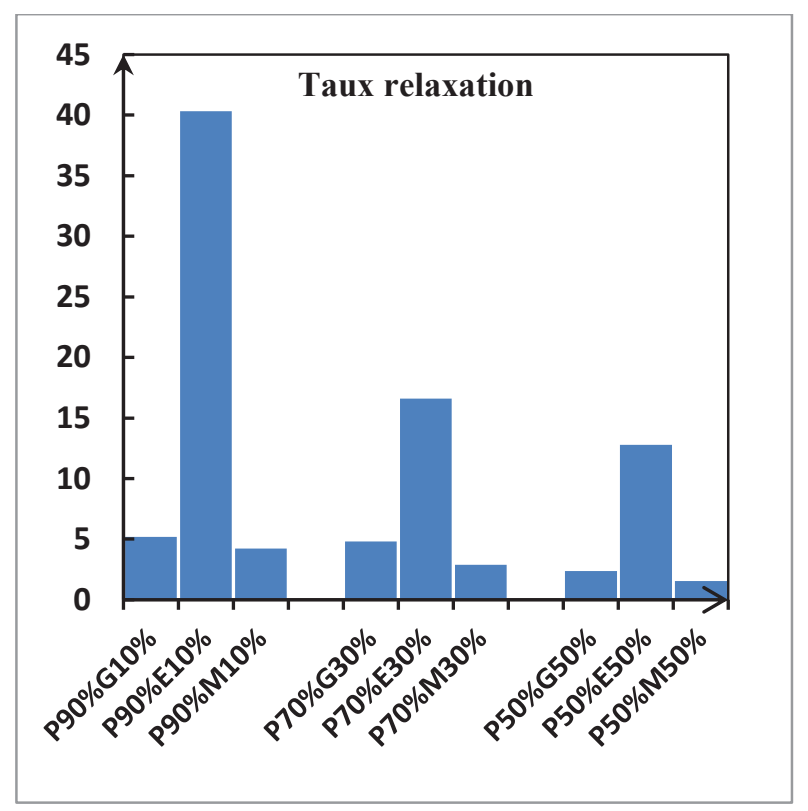

Fig.4. Taux de relaxation des briquettes

Les briquettes fabriquées avec la gomme arabique présentent un taux de relaxation qui va de $5,2 \%$ à $2,38 \%$ en fonction de la proportion des matières premières. La P90\%G10\% a le taux le plus élevé $(5,2 \%)$ et le petit (2,38\%) est la P50\%G50\%. On constate que le taux de relaxation diminue avec la quantité de gomme utilisée.

Les briquettes fabriquées avec l'eau comme liant semblent être les briquettes qui se relaxent le plus; sa relaxation varie de $12,7 \%$ à 40,32\%. La P90\%E10\% a le taux le plus élevé et la $\mathrm{P} 50 \% \mathrm{E} 50 \%$ a le plus petit avec un taux de relaxation de $12,7 \%$. Gado et al., ont trouvé un taux de relaxation de $12,3 \%$ des briquettes de déchets papiers.

Les briquettes fabriquées avec la mélasse ont un taux de relaxation qui varie très peu (4,23-1,53\%). La P90\%M10\% $(4,23 \%)$ a un taux plus grand que celui de la briquette P50\%M50\% (1,53\%). Les résultats obtenus avec les trois types de briquettes nous permettent de dire que les liants ont une influence sur leur cohésion. Les briquettes fabriquées avec la mélasse présentent le plus faible taux de relaxation parce que ces dernières sont plus cohérentes au démoulage. Celles fabriquées avec la gomme arabique est élevé parce qu'elles semblent être moins cohérentes au démoulage.

\subsubsection{La masse volumique}

La masse volumique des trois types de briquettes varie de $0,879 \mathrm{~g} / \mathrm{cm}^{3}$ à $0,54 \mathrm{~g} / \mathrm{cm}^{3}$ et les résultats ont été regroupés dans la figure 5 .

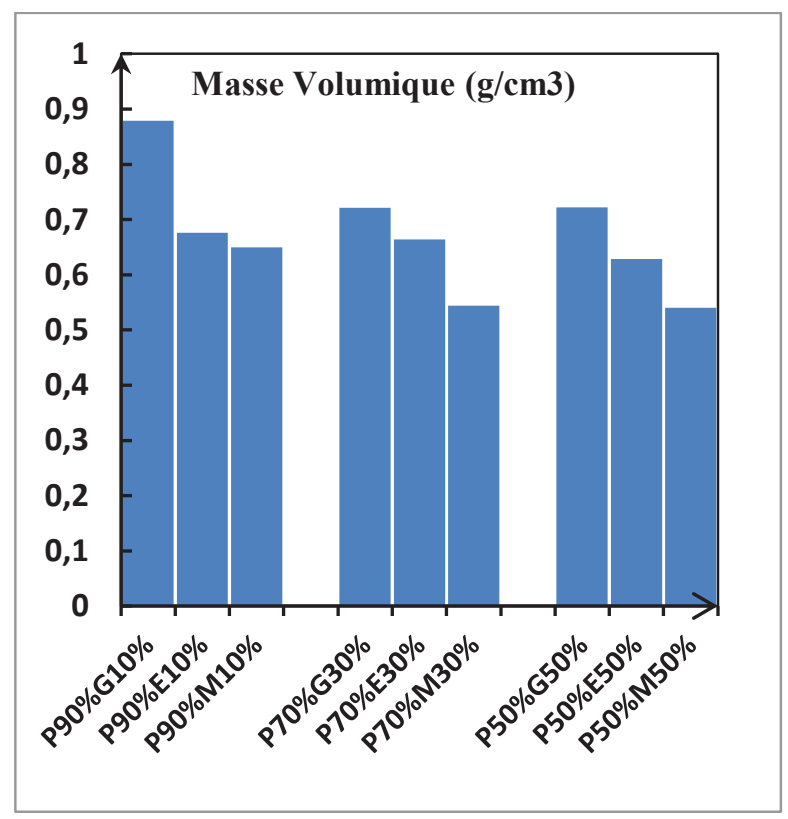

Fig.5. Masse volumique des briquettes

Les briquettes fabriquées avec la gomme arabique ont de grande masse volumique. La P90\%G10\% est celle la plus élevé $\left(0,879 \mathrm{~g} / \mathrm{cm}^{3}\right)$ et P50\%G50\% la plus basse $(0,721$ $\left.\mathrm{g} / \mathrm{cm}^{3}\right)$. Elle évolue en fonction de la proportion des matières premières des briquettes. Les briquettes fabriquées avec la mélasse ont une masse volumique relativement petite par rapport aux autres liants étudiés. La P90\%M10 a une masse volumique de $0,649 \mathrm{~g} / \mathrm{cm}^{3}$ et la $\mathrm{P} 50 \% \mathrm{M} 50 \%$ à la plus petite masse volumique $0,54 \mathrm{~g} / \mathrm{cm}^{3}$.

Les briquettes fabriquées avec l'eau ont une masse volumique qui varie très peu $\left(6,7-6,2 \mathrm{~g} / \mathrm{cm}^{3}\right)$. La $\mathrm{P} 90 \% \mathrm{E} 10 \%$ a la plus grande masse volumique $\left(6,7 \mathrm{~g} / \mathrm{cm}^{3}\right)$ et $\mathrm{P} 50 \% \mathrm{E} 50 \%$ la plus petite $\left(6,2 \mathrm{~g} / \mathrm{cm}^{3}\right)$. D'autres auteurs tels que I. H. GADO et al (2013) ont trouvé une masse volumique de $0,54 \mathrm{~g} / \mathrm{cm}^{3}$ pour le compactage des papiers de bureau. A. Olorunnisola (2007) a obtenu une masse 
volumique de $9,5 \mathrm{~g} / \mathrm{cm}$ pour le compactage de papier $(75 \%)$ mélangé aux noix de coco (25\%). Ces auteurs ont utilisé le l'eau comme liant et la pression était de 60 bars. Suite aux résultats de toutes ses briquettes obtenus, nous constatons que la gomme arabique est le liant qui a la masse volumique la plus élevé avec la P90\%G10\%. Cela peut s'expliquer par le fait que lors de la densification la gomme arabique ne s'échappe pas comme dans le cas de l'eau ou la mélasse. La gomme arabique a une granulométrie beaucoup plus élevé que les autres liants utilisés donc elle s'échappe en moindre quantité.

\subsubsection{L'Indice de Résistance à l'Impact}

L'indice de résistance à l'impact d'une briquette est l'un des principaux paramètres qui décrivent la solidité du biocombustible d'où sa qualité physique. La figure 6 illustre les résultats de la détermination de l'indice de résistance à l'impact des briquettes.

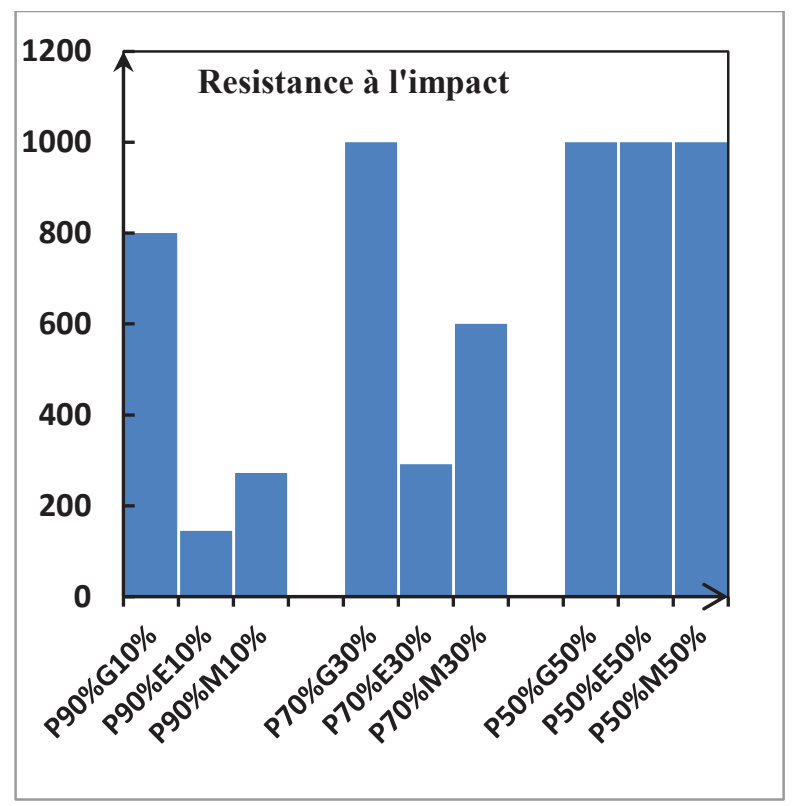

Fig.6. Indice de Résistance à l'impact des briquettes

Les briquettes P90\%G10\% ont un IRI de 800, celles $\mathrm{P} 70 \% \mathrm{G} 30 \%$ et $\mathrm{P} 50 \% \mathrm{G} 50 \%$ ont un IRI de 1000 . La gomme arabique permet donc d'élever l'indice de résistance à l'impact des briquettes. Les briquettes P90\%E10\% ont un IRI de 144, celles P70\%GE0\% de 291 et P50\%E50\% ont un IRI de 1000. L'eau permet donc de solidifier la briquette. Les briquettes P90\%M10\% ont un IRI de 272 , celles $\mathrm{P} 70 \% \mathrm{M} 30 \%$ de 600 et $\mathrm{P} 50 \% \mathrm{M} 50 \%$ ont un IRI de 1000. On pourrait donc dire que la mélasse impact sur la solidité des briquettes de combustibles. Tous les liants utilisés dans cette étude ont un impact positif sur la qualité physique des briquettes. D'après Richards (1990), les briquettes de combustibles sont acceptables lorsque leurs indices de résistance à l'impact est supérieur à 50. Dans l'ensemble, nous constatons que toutes les briquettes fabriquées ont un indice de résistance à l'impact acceptable. Toutefois nous constatons qu'avec les trois types de liants et pour les proportions de 50\% de liant l'IRI avoisine une valeur de 1000 . Ce qui signifie que quel que soit le liant que nous avons utilisé l'IRI croît avec sa proportion. Et c'est dans les mélanges à base de gomme arabique que l'on trouve les plus grandes valeurs d'IRI quel que soit ratio du mélange.

\subsubsection{Le taux d'humidité}

La Figure 7 montre les résultats obtenus suite à l'analyse du taux d'humidité des 9 types de briquettes et leurs matières premières.

Toutes les briquettes présentent un taux d'humidité qui varie entre $8-15 \%$. Nous constatons que les briquettes fabriquées avec la gomme arabique ont un faible taux d'humidité et cela peut s'expliquer par le fait que les matières premières de ses briquettes, la gomme arabique $(12,29 \%)$ et les déchets papiers $(7,28 \%)$, ont un taux d'humidité relativement faible. Parmi ses briquettes, la P90\%G10\% a le taux d'humidité le plus faible $(8,44 \%)$ et la $\mathrm{P} 50 \% \mathrm{G} 50 \%$ est celle qui a le taux le plus élevé $(11,49 \%)$. Par contre, les briquettes fabriquées avec la mélasse (jusqu'à 15,1\%) ont un taux un peu plus élevé parce que la mélasse elle-même a un taux d'humidité de 26,95\%. La briquette P50\%M50\% est celle qui a le taux d'humidité la plus élevée et cela est dû au fait que c'est elle qui a la plus grande quantité de mélasse. Les briquettes fabriquées avec l'eau comme liant à un taux d'humidité presque stable $(8,1-9,1 \%)$. Cela peut s'expliquer par le fait qu'une grande quantité d'eau est évacuée après 1 ' 'exposition des briquettes à l'air libre pour séchage. Les déchets papiers/cartons ont un taux d'humidité d'environ $7,3 \%$.

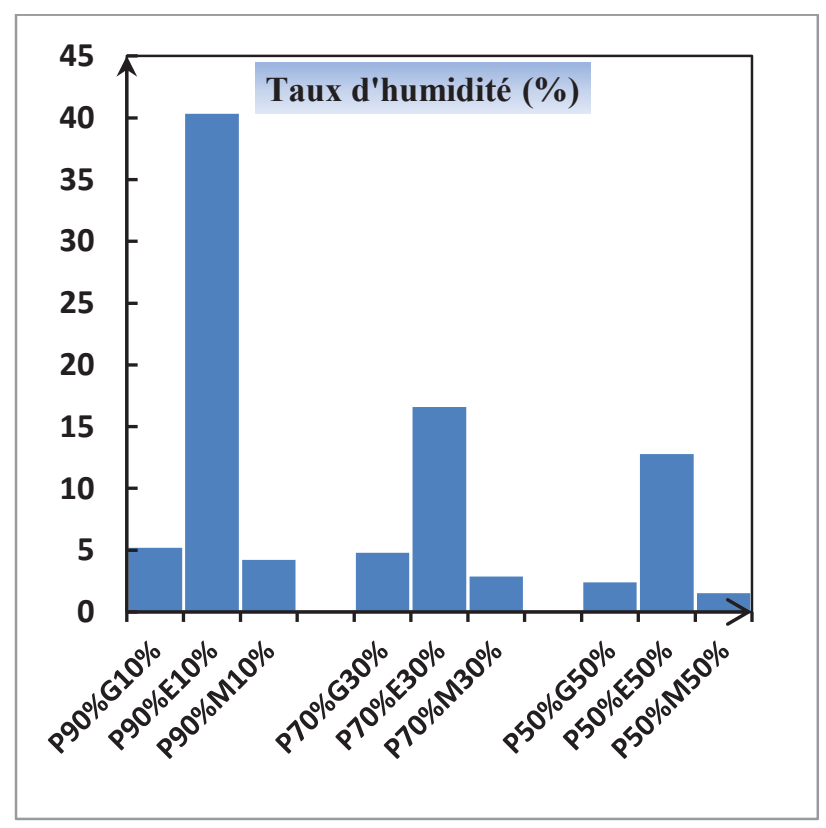

Fig.7. Taux d'humidité des briquettes et des matières premières 


\subsubsection{Le Pouvoir Calorifique Inferieur}

Les résultats de PCI pour les briquettes sont de l'ordre de 12,91-14,3 MJ/kg et présentés dans la figure 8 .

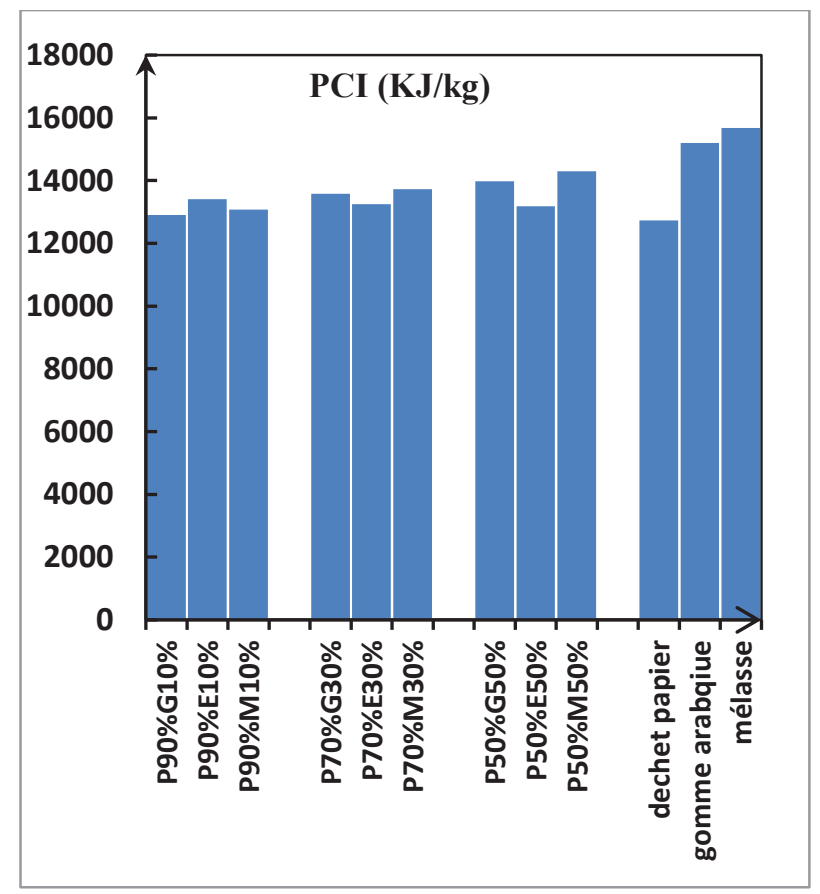

Fig.8. PCI des briquettes et des matières premières

La gomme arabique a un PCI de $15,2 \mathrm{MJ} / \mathrm{kg}$ et les briquettes fabriquées $\quad \mathrm{P} 90 \% \mathrm{G} 10 \% \quad \mathrm{P} 70 \% \mathrm{G} 30 \%$ P50\%G50\% respectivement $12,91 \mathrm{MJ} / \mathrm{kg} 13,58 \mathrm{MJ} / \mathrm{kg}$ et $13,98 \mathrm{MJ} / \mathrm{kg}$. Le PCI croissant en fonction des proportions de gomme arabique ajoutée dans la composition des briquettes de combustibles. La mélasse a un PCI de 15,68 $\mathrm{MJ} / \mathrm{kg}$ et les briquettes fabriquées $\mathrm{P} 90 \% \mathrm{M} 10 \%$ P70\%M30\% P50\%M50\% respectivement 13,081 MJ $/ \mathrm{kg}$ $13,75 \mathrm{MJ} / \mathrm{kg}$ et $14,3 \mathrm{MJ} / \mathrm{kg}$. Nous constatons que la mélasse en plus d'être un bon liant pour les briquettes est aussi un bon apport en matière d'énergie pour les briquettes. Les briquettes fabriquées avec l'eau ont un PCI qui est environ $13 \mathrm{MJ} / \mathrm{kg}$ pour toutes les proportions d'eau expérimentées. S. Yaman et al. (2000) dans leurs travaux ont trouvé que le PCI des briquettes à base de papiers est de 13,0 MJ/kg. De tout ce qui précède, nous pouvons remarquer que les briquettes fabriquées avec la mélasse ont les meilleurs PCI. Compte tenu du fait que la mélasse à un PCI élevé, elle augmente le PCI des briquettes lorsqu'elle est utilisée dans la fabrication des briquettes.

\subsubsection{Le taux de matières volatiles}

La figure 9 donne les taux de matières volatiles des briquettes étudiées qui varient entre $70-82 \%$.

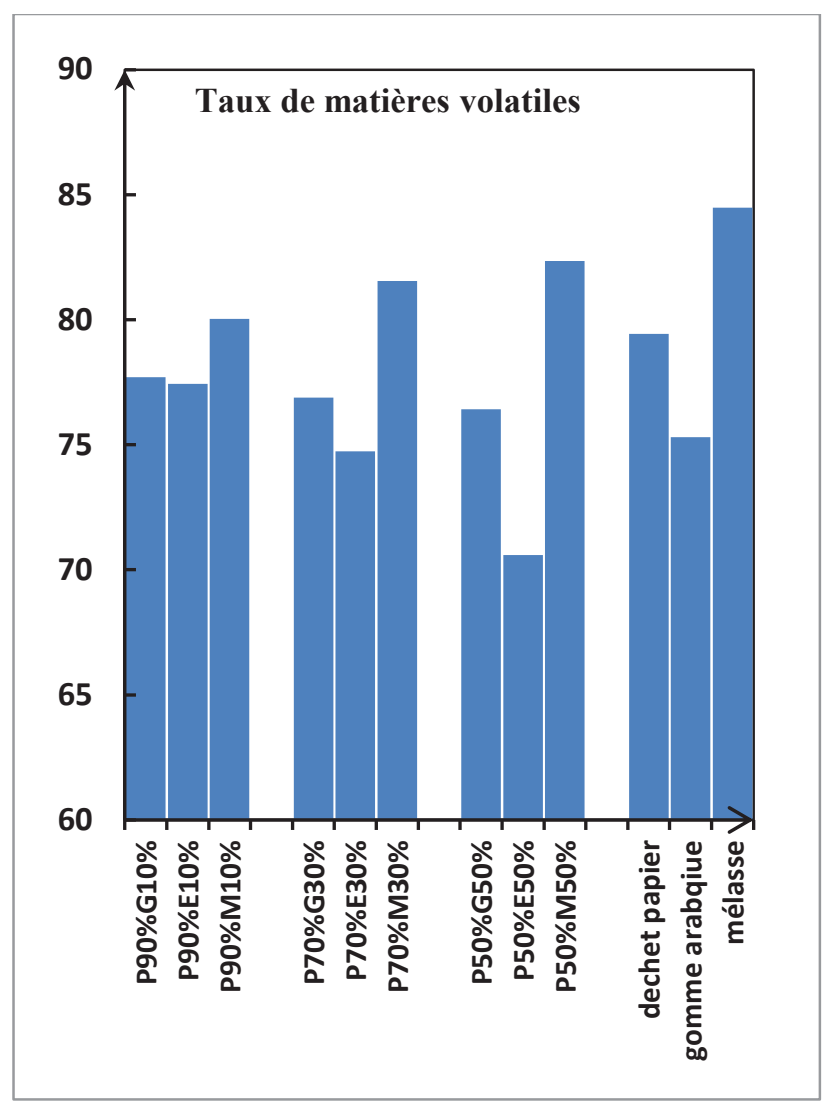

Fig.9. Taux de matières volatiles

La gomme arabique a un taux de matières volatiles d'environ $75,29 \%$. Pour les briquettes fabriquées avec la gomme arabique, la $\mathrm{P} 90 \% \mathrm{G} 10 \%$ a le taux de cendres le plus élevé $(77,7 \%)$ et la P $50 \% \mathrm{G} 50 \%$ a le taux de MOV le plus faible $(76,42 \%)$. Le constant fait est que la gomme arabique réduit le taux de matières volatiles des briquettes, ce qui implique que la gomme arabique permet d'avoir un combustible moins fumant. Pour les briquettes fabriquées avec l'eau, la $\mathrm{P} 90 \% \mathrm{E} 10 \%$ possède un taux de $77,43 \%$ et la P $50 \% \mathrm{E} 50 \%$ a un taux de $70,59 \%$. Nous constatons que le taux de matières volatiles des briquettes varie en fonction des proportions des matières premières de la briquette. Les briquettes fabriquées avec la mélasse ont un taux de matières volatiles élevé. La $\mathrm{P} 90 \% \mathrm{M} 10 \%$ a un taux de $80,04 \%$ et la $\mathrm{P} 50 \% \mathrm{M} 50 \%$ a un taux de $82,34 \%$. Cela s'explique par le fait que la mélasse elle-même a un taux de $84,48 \%$. Plus le taux de mélasse dans la composition de la briquette croît, plus son taux de matières volatiles s'accroit. 


\subsubsection{Le taux de cendres}

La figure 10 montre les taux de cendres des briquettes étudiées et leurs matières premières qui sont entre 2,36$11,85 \%$.

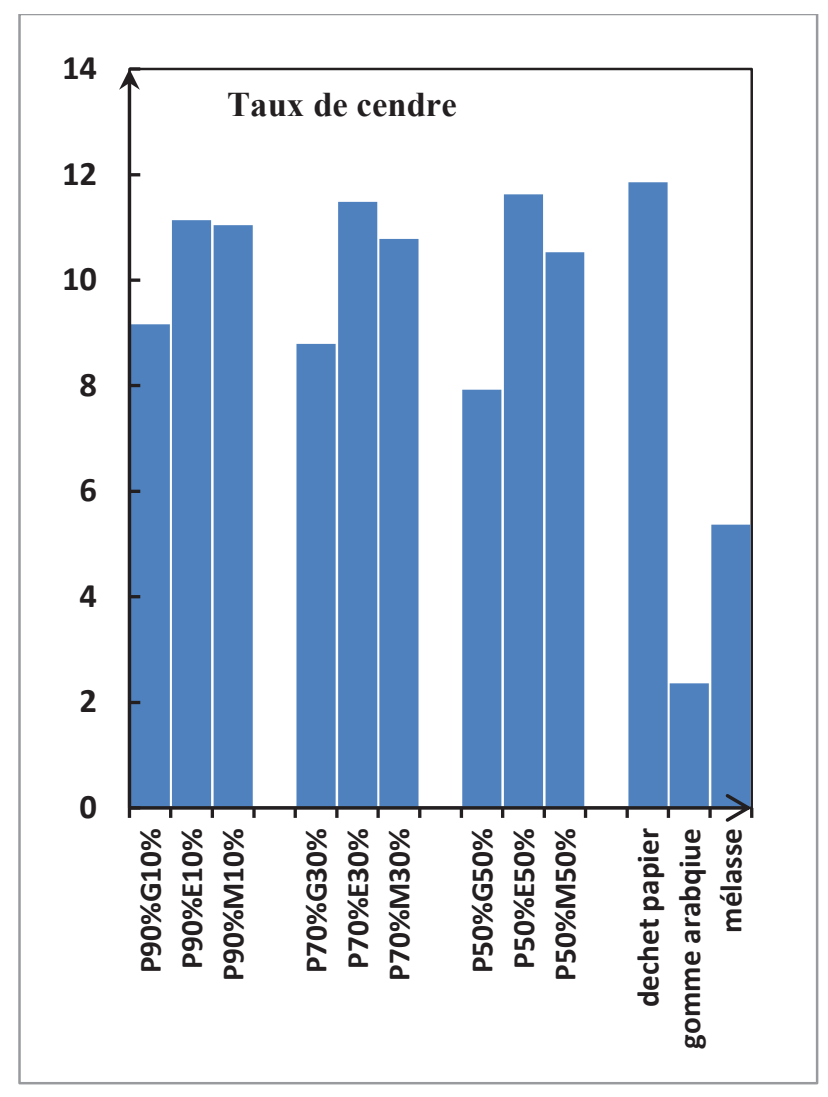

Fig.10. Taux de cendres des briquettes

La gomme arabique a un taux de cendres de 2,36\%, cela s'explique par le fait que la gomme arabique est riche en matières minérales. Les briquettes fabriquées avec la gomme arabique ont un taux qui varie entre 7,92-9,16\%, la $\mathrm{P} 90 \% \mathrm{G} 10 \%$ a le plus grand taux $(9,1 \%)$ et la $\mathrm{P} 50 \% \mathrm{G} 50 \%$ a le plus faible taux $(7,92 \%)$. Nous remarquons que les proportions de gomme arabique utilisées dans les briquettes diminuent avec le taux de cendres des briquettes. Les briquettes fabriquées avec l'eau ont un taux de cendres qui varie très peu $(11,13$ $11,62 \%)$. La P90\%E10\% a le plus petit taux (11,13\%) et la $\mathrm{P} 50 \% \mathrm{E} 50 \%$ a le plus grand taux $(11,62 \%)$. L'ajout d'eau dans les briquettes augmente de très peu le taux de cendres.

La mélasse a un taux de cendres de 5,36\%. Les briquettes fabriquées avec la mélasse ont un taux de cendres qui varie en fonction des proportions des matières premières. La $\mathrm{P} 90 \% \mathrm{M} 10 \%$ a le grand taux de cendres $(11,04 \%)$ et la $\mathrm{P} 50 \% \mathrm{M} 50 \%$ a le plus faible taux $(10,53 \%)$. On constate que, de toutes les briquettes étudiées, celles à base de mélasse conduisent aux taux de cendre les plus bas. Cela peut s'expliquer par le fait que la mélasse se compose d'un taux de matières volatiles nettement plus élevés que respectivement ceux de l'eau et de la gomme arabique.

\subsubsection{Le taux de carbone fixe}

La figure 11 montre le taux de carbone fixe de toutes les briquettes fabriquées et leurs matières premières.

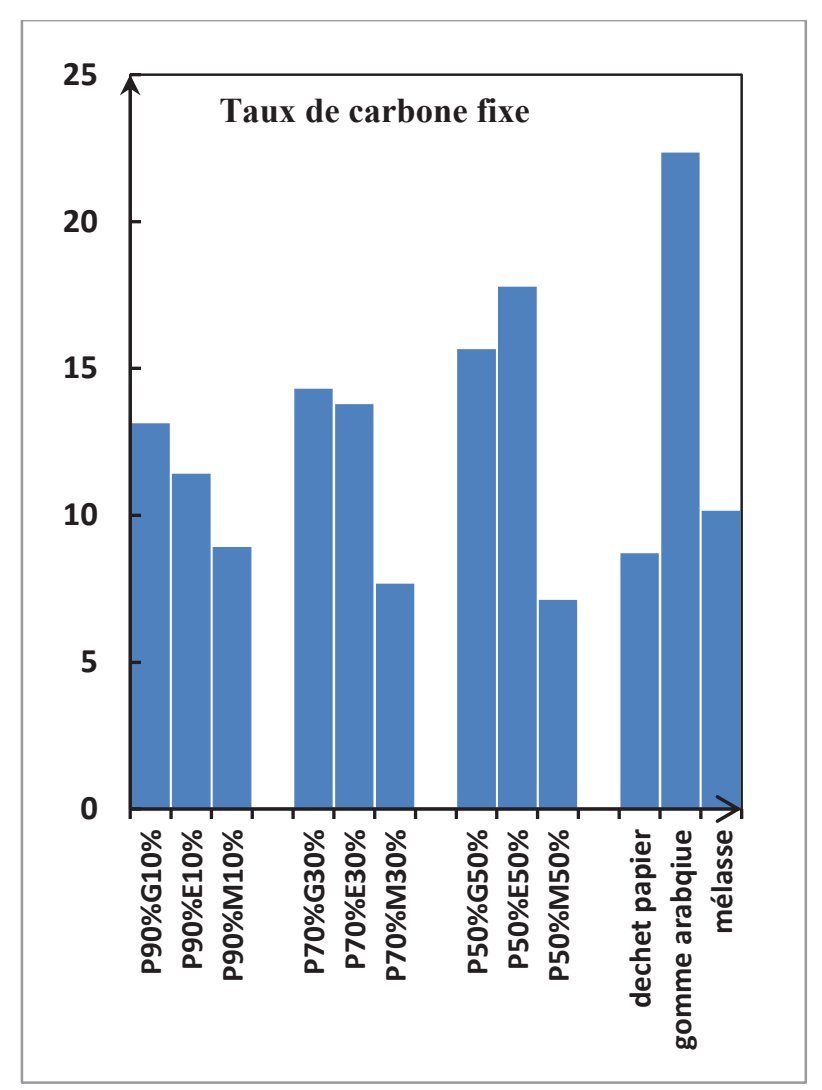

Fig. 11. Taux de carbone fixe des briquettes

La gomme arabique a un taux de carbone fixe de $22,34 \%$. Les briquettes fabriquées avec la gomme ont un taux de carbone fixe élevé, la $\mathrm{P} 90 \% \mathrm{G} 10 \%$ a le taux le plus faible $(13,13 \%)$ et la P50\%G50\% le taux le plus élevé. Le taux de carbone fixe des briquettes augmente avec l'ajout de la gomme arabique. Les briquettes fabriquées avec l'eau ont un taux de carbone fixe qui varie entre $11,42-17,77 \%$. Les proportions d'eau dans les mélanges pour la fabrication de briquettes ont un impact donc sur leurs taux de carbone fixe. La mélasse a un taux de carbone fixe de 10,14\%. Les briquettes fabriquées avec la mélasse ont un taux qui est peu élevé, la $\mathrm{P} 90 \% \mathrm{M} 10 \%$ a le plus grand taux $(8,91 \%)$ et la $\mathrm{P} 50 \% \mathrm{M} 50 \%$ le plus faible $(7,12 \%)$. Le taux de carbone fixe des briquettes diminue avec l'ajout de la mélasse.

À l'issue des résultats, nous pouvons dire que les liants ont une influence sur les caractéristiques physique, chimique et mécanique des briquettes de combustibles. Parmi les liants utilisés dans cette étude, nous constatons que la mélasse est le meilleur liant qui offre un faible taux de relaxation. Avec ce liant, toutes les briquettes fabriquées ont un taux de relaxation de moins de $5 \%$. Elle est suivie de très près par la gomme arabique qui a un taux de relaxation intéressant également. L'eau quant à elle a un taux qui va jusqu'à $40 \%$. S'agissant de la masse volumique des briquettes, la mélasse est encore meilleure par rapport 
à la gomme arabique et à l'eau car elle a une faible masse volumique. La gomme a une masse volumique qui varie peu lorsqu'elle est utilisée dans le compactage des papiers bureaux. Lorsque les papiers bureaux sont mélangés avec l'eau pour être compactés, ils ont une masse volumique inférieure à $0,9 \mathrm{~g} / \mathrm{cm}^{3}$. Pour la durabilité de la briquette, la gomme arabique est le meilleur liant par rapport aux autres étudiés. A partir de $30 \%$ de mélange de la gomme, la briquette tend à avoir un IRI de plus de 1000. La mélasse rend la briquette rigide parce qu'elle a un IRI de plus de 50, alors c'est un bon liant. Les briquettes fabriquées avec l'eau ont un IRI acceptable. Les briquettes fabriquées avec la gomme ont un taux d'humidité qui oscille entre 8-11\% par contre celles avec la mélasse varie entre 8-15\%. Le taux d'humidité de la gomme arabique (12,29\%) est deux fois plus petit que celui de la mélasse $(26,95 \%)$ et que les papiers bureaux ont un taux d'humidité d'environ 7,3\%. Cela explique pourquoi le taux d'humidité des briquettes augmente avec les proportions de liants utilisées dans le compactage des déchets papiers bureaux. Les briquettes fabriquées avec la gomme arabique ont un taux de matières volatiles élevé par rapport à celles fabriquées avec la mélasse. Le taux de matières volatiles des déchets papiers obtenu est d'environ $79,44 \%$, celui de la gomme arabique $75,29 \%$ et la mélasse $84,48 \%$. Les matières premières de nos briquettes étudiées ont un faible taux de cendres. La gomme arabique a un taux de cendres de 2,36\%, la mélasse $5,36 \%$ et les papiers bureaux environ $11,85 \%$. Ces faibles taux expliquent les résultats obtenus pour les briquettes. La quantité de déchets papiers/cartons croit avec celle du taux de cendres. Le taux de carbone fixe obtenu est la conséquence des résultats du taux de matières volatiles et $\mathrm{du}$ taux de cendres. Nous remarquons que le taux de cendres de la mélasse et celui de la gomme arabique varient très peu dans toutes les proportions. La mélasse (15,68\%) a un PCI élevé par rapport à la gomme arabique $(15,2 \%)$. Les briquettes fabriquées avec la mélasse ont les PCI les plus élevés par rapport à celles fabriquées avec la gomme arabique. Il faut noter aussi qu'avec ces deux liants on obtient de meilleurs résultats par rapport à l'eau.

\section{CONCLUSION ET PERSPECTIVES}

Au terme de notre étude, il est à noter que les briquettes issues des déchets papiers/cartons peuvent être utilisées en lieu et place des bois de chauffe et sont compatibles avec les foyers habituellement utilisés dans les ménages pour trois raisons essentielles. Tout d'abord ces briquettes ont un pouvoir calorifique acceptable. Ensuite l'utilisation des briquettes va permettre d'utiliser une autre source d'énergie alternative et va enfin contribuer à la protection de l'environnement menacé aujourd'hui par le phénomène des gaz à effet de serre. A l'issue des résultats physique thermique et mécanique, les conclusions suivantes peuvent être tirées :
- la mélasse est le meilleur liant pour la cohérence des briquettes ce qui rend la briquette stable;

- la mélasse est le meilleur liant pour une faible masse volumique des briquettes ;

- la mélasse permet d'avoir un meilleur IRI des briquettes, elle assure une meilleure durabilité des briquettes ;

- les briquettes fabriquées avec l'eau présentent un faible taux d'humidité par rapport à celles fabriquées avec la gomme arabique et la mélasse ;

- les briquettes fabriquées avec la gomme arabique possède le plus petit taux de matières volatiles ;

- les briquettes fabriquées avec la mélasse et l'eau ont un taux de cendres qui varie très peu;

- les briquettes fabriquées avec l'eau ont le plus grand taux de carbone fixe ;

- les briquettes de combustibles (retenues comme meilleures) ont environ un PCI deux fois inférieur au PCI du charbon du bois (30 MJ/kg); il s'agit de P50\%G50\% $\mathrm{P} 50 \% \mathrm{E} 50 \%$ et $\mathrm{P} 50 \% \mathrm{M} 50 \%$;

- les briquettes fabriquées avec la mélasse présentent le meilleur PCI comparativement aux autres.

Ce travail bien qu'intéressant ne nous permet pas d'affirmer laquelle des briquettes est la meilleure par rapport à l'autre. Des tests supplémentaires sont nécessaires pour arriver à une telles conclusion. C'est la raison pour laquelle des perspectives sont proposées pour une continuité éventuelle de cette étude. Il s'agit de faire des tests d'incinération pour observer le comportement des différentes briquettes dans des conditions proches de leur utilisation dans les foyers de cuisine ou dans les chaudières industrielles et semi industrielles. Au cours de ces tests avec différents paramètres de combustion, nous analyserons les taux d'émission polluants gazeux tels que les oxydes d'azote et de carbone, le dioxyde de soufre, le dioxygène et le taux de cendre. Nous ferons un suivi des températures de combustion en phase gazeuse. Outre cette étude il est également prévu de :

- étudier les paramètres énergétiques des briquettes (indice d'inflammation, rendement de combustion, consommation horaire en phase flamme et consommation horaire en phase braise) ;

- étudier la faisabilité des briquettes à base de déchets papiers/cartons afin de les comparer au charbon de bois ;

- tester d'autres liants tels que l'amidon et la farine pour augmenter le PCI, la durabilité et la masse volumique des briquettes.

\section{REFERENCES BIBLIOGRAPHIQUES}

ADEME, Critt Bois, Fibois, \& CTBA, Mesure des caractéristiques des combustibles bois, 2001. 
Al-Widyan, M. I., Al-Jalil, H. F., Stress-density relationship and energy requirement of compressed olive cake. Appl. Eng. Agric. 17, 749-753, 2001.

BEMB, G. C, Le traitement des ordures ménagères et l'agriculture urbaine et périurbaine dans la ville de Bertoua, Cameroun : Institut national de la jeunesse et des sports-Conseiller Principal de Jeunesse et d'Animation, 2009.

Chin, O.C., Siddiqui, Kamal. M., Characteristics of some biomass briquettes prepared under modest die pressures. Biomass. Bio-energy.18, 223-228 (2000).

Gado, I. H., Ouiminga, S. K., Daho, T., Yonli, A. H., Sougoti, M., and Koulidiati, J. characterization of briquettes coming from compaction of paper and cardboard waste at low and medium pressures, ISSN 1877-2641, Waste and Biomass Valorization, volume 4number 4- December 2013.

Khankari KK, Shrivastava M, Morey RV. Densification characteristics of rice hulls. ASAE paper no. 89-6093. St.Joseph, MI: ASABE ; 1989.

Lindley JA, Vossoughi M. Physical properties of biomass briquettes. Transactions of the ASAE 1989 ; 32 : 361-6.

Marie Sandrine DUSABE, Etude de faisabilité technique et financière de la valorisation des déchets ménagers organiques papiers et cartons pour la fabrication des briquettes combustibles à Bujumbura, Mémoire de master II, 2ie, Burkina.

Mélanie PROUST, Campagne de caractérisation des ordures ménagères de la Ville de Ouagadougou,2001.
Njenga M., Karanja N., Prain G., Malii J., Munyao P., Gathuru, K., et al. (2009). Community-based energy Briquette production from urban orgnic waste at Kahawa Soweto. Informal settlement, Nairobi. ISSN 1811-1440, Urban Harvest.

Norme AFNOR/X 34 B. N-287.

Raghavan JK, Conkle HN. Physical characteristic measurements for reconstituted coal pellets. Proceeding of the Institute for Briquetting and Agglomeration (IBA) 1991; $22: 85-96$.

Richards, S.R.: Physical testing of fuel briquettes. Fuel. Process.Technol.25 (2), 89-100 (1990).

Sah P, Singh B, Agrawal U. Compaction behavior of straw. Journal of Agricultural Engineering - India 1980; $18: 89-96$

Shrivastava, M., Shrivastava, P., Khankari, KK. : Densification characteristics of rice husk under cold and hot compression. In: Dodd, VA., Grace, PM. (eds) Agricultural Engineering: proceedings of the 11th international congress on agricultural engineering, Dublin, 4-8 Sept 1989. Rotterdam: A.A. Balkema Pub; 1989. p. 2441-2443.

Yaman S, M. Sahan, H. Haykiri-acma, K. Sesen and S. Kucukbayrak, Production of fuel.

briquettes from olive refuse and paper mill waste. Fuel Processing Technology 68 : 23-31, 2000. 


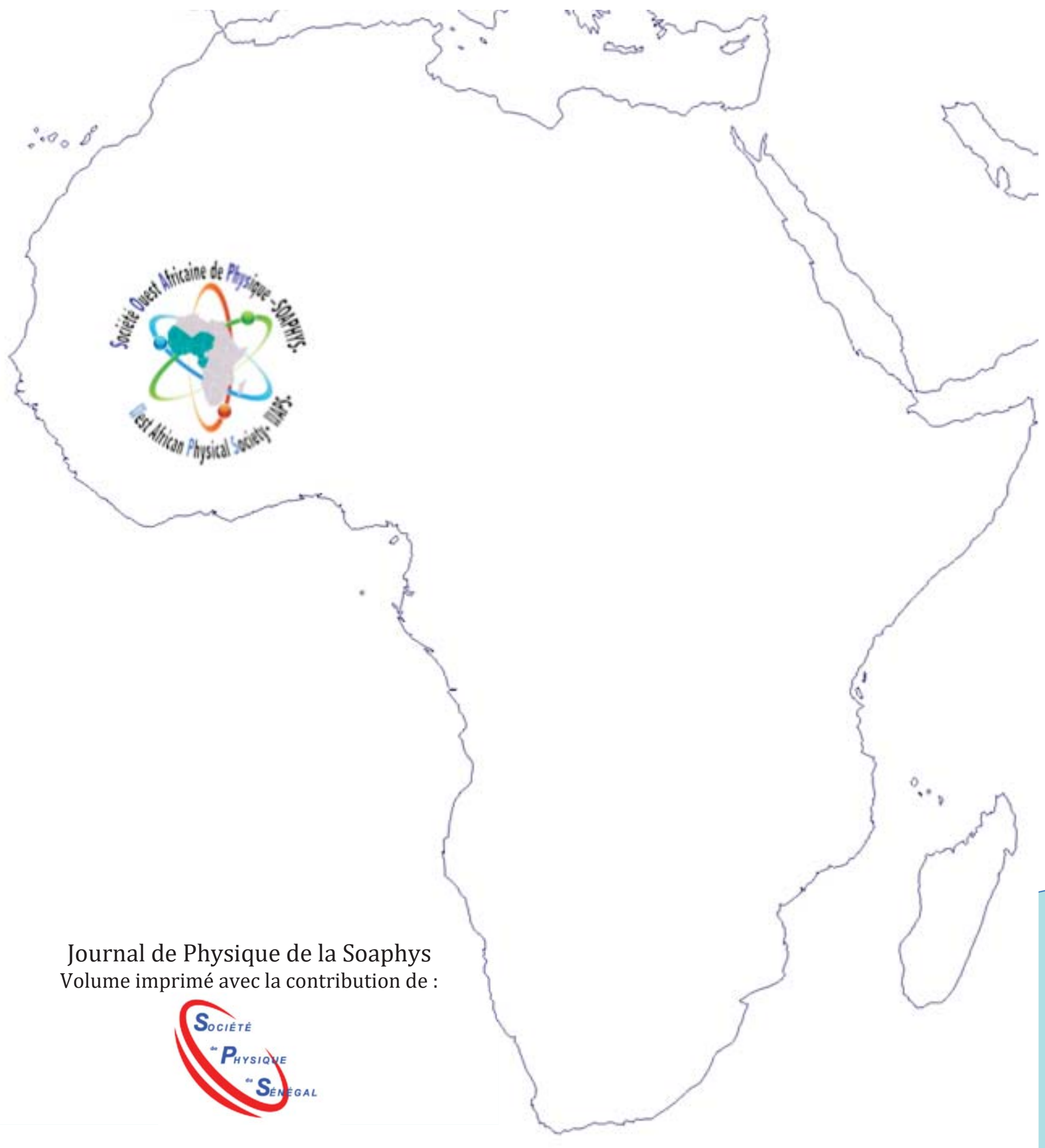

